\title{
Cinco años de experiencia con el agar de capa delgada para el diagnóstico rápido de tuberculosis
}

\author{
Gloria Isabel Mejía 1,2, Angela Guzmán ${ }^{1}$, Carlos Andrés Agudelo 2, Hugo Trujillo 1 , \\ Jaime Robledo ${ }^{1,2}$ \\ ${ }^{1}$ Unidad de Bacteriología y Micobacterias, Corporación para Investigaciones Biológicas (CIB), Medellín, \\ Colombia. \\ 2 Facultad de Medicina, Universidad Pontificia Bolivariana, Medellín, Colombia
}

La tuberculosis continúa siendo un problema de salud pública en el mundo, principalmente en los países en vía de desarrollo, donde ocurre el $95 \%$ de los casos. Estos países no tienen fácil acceso a los métodos de diagnóstico rápido actualmente disponibles, debido al alto costo que tienen. Por tanto, se hace necesario el desarrollo de técnicas rápidas de más bajo costo que sean accesibles a estas regiones. Este trabajo tuvo como objetivo comparar un medio de cultivo rápido y de bajo costo, el agar de capa delgada (CD7H11), con el medio de LowensteinJensen. Para esto se procesaron 1.809 muestras clínicas de pacientes con diagnóstico presuntivo de tuberculosis y que requirieron cultivo. Las muestras se procesaron según los métodos estándar recomendados y se sembraron en ambos medios de cultivo. Se comparó la rapidez para la detección de cultivos positivos y la sensibilidad, la especificidad, los valores predictivos (VPP y VPN) y la concordancia de CD7H11 con respecto al método de referencia. CD7H11 mostró una sensibilidad del 73,5\% (IC95\%:69,6-80,4) y una especificidad del 99,2\% (IC95\%: 98,8-99,6), valor predictivo positivo de 90,4\% (IC95\%: 85,3-95,6) y valor predictivo negativo de 97,6\% (IC95\%: 96,8-98,3). La concordancia entre los dos métodos fue de 0,52 (coeficiente kappa). CD7H11 tuvo un tiempo promedio para la detección de las micobacterias de 11 días $(\mathrm{DE} \pm 4,9)$, frente a 26,5 $(\mathrm{DE} \pm 8.6)$ días del LJ. Se obtuvieron resultados similares al comparar las muestras pulmonares y multibacilares. CD7H11 mostró una sensibilidad menor en muestras extrapulmonares, comparada con las pulmonares (65,8; IC95\%: 55,1-76,5 vs. 81,0; IC95\%: 72,4-89,7). El uso concomitante de los medios de cultivo aumentó la sensibilidad pues $24,1 \%$ de las muestras se detectaron sólo por $\mathrm{LJ}$ y $7,1 \%$ por $\mathrm{CD} 7 \mathrm{H} 11$. El medio de capa delgada demostró ser un método rápido de cultivo para micobacterias que es fácilmente accesible a los sistemas de salud de los países en desarrollo, en conjunto con los métodos tradicionales mejora el desempeño en el diagnóstico de la tuberculosis.

Palabras clave: tuberculosis, diagnóstico, cultivos, capa delgada, diagnóstico rápido de tuberculosis.

Five year experience with thin layer agar medium for rapid diagnosis of tuberculosis

Tuberculosis represents a public health problem worldwide, mainly in developing countries where $95 \%$ of the cases occur. New technologies that support rapid diagnosis are not available in these settings because of high cost. New, rapid, and less expensive techniques are necessary before diagnosis can be improved in these areas. The present work compared the performance of a rapid and costly culture media, thin layer agar (CD7H11), with the traditional LowensteinJensen (LJ) culture method. For this comparison, 1,809 clinical specimens were processed for diagnosis of mycobacterial infections. Clinical samples were processed according to standard procedures and cultured concomitantly in LJ and CD7H11. The times required to obtain an isolate were compared for culture media. Sensitivity (S), specificity (Sp), predictive values (PPV, NPV) and agreement (kappa coefficient) were calculated for CD7H11, with LJ serving as the gold standard. CD7H11 showed S to be $73.5 \%$ (C.I.95\%: 69.6-80.4), Sp to be $99.2 \%$ (C.I.95\%: 98.8-99.6), PPV 90.4\% (C.I.95\%: 85.3-95.6) and NPV 97.6\% (C.I.95\%: 96.8-98.3). Agreement had a kappa coefficient of 0.52 . The mean time for $\mathrm{CD} 7 \mathrm{H} 11$ was 11 days $(\mathrm{SD} \pm 4.9)$ compared 
with $26.5(S D \pm 8.6)$ days for LJ. Similar results were obtained in a comparison of respiratory and multibacillary clinical samples. In extrapulmonary samples and those with lowered bacillus count, CD7H11 demonstrated a lower sensitivity. The concomitant use of both culture media enhanced sensitivity of detection. CD7H11 proved a simple and rapid technique for culturing mycobacteria and can be combined with traditional methods for improving laboratory capability for diagnosis of tuberculosis.

Key words: $M$. tuberculosis culture, thin layer $7 \mathrm{H} 11$ culture media, rapid diagnosis of tuberculosis

La tuberculosis continúa siendo un problema importante de salud pública en el mundo, aun en aquellos países en los cuales se creía controlada. $\mathrm{EI} \mathrm{VIH} /$ sida, la multirresistencia y el deterioro de los programas de vigilancia y control en muchas áreas han hecho de esta enfermedad un problema creciente que se ha visto reflejado en las últimas cifras reportadas por la Organización Mundial de la Salud (OMS), 1,5 millones de muertos por año, 8 millones de casos nuevos y un tercio de la población mundial infectada (1).

Uno de los pilares en el control de la enfermedad es mejorar la oportunidad del diagnóstico y la identificación; sin embargo, los medios de cultivo más usados y tradicionales (Lowenstein-Jensen y Ogawa) requieren tres semanas en promedio para la detección del crecimiento de micobacterias y otras 3-4 semanas para obtener los resultados de las pruebas de sensibilidad a los medicamentos (2). Los medios de cultivo líquidos logran un crecimiento más rápido con mayor sensibilidad y se recomienda su uso en conjunto con medios sólidos para el diagnóstico rutinario de tuberculosis. No obstante, no permiten la identificación rápida de la especie de micobacteria si no están acompañados de un medio sólido o de la utilización de sondas de ADN (3). Este panorama ha generado la aparición de múltiples técnicas microbiológicas, radiométricas y moleculares cuya utilización permite disminuir de una manera importante el tiempo necesario para el diagnóstico; aunque estas técnicas, por su alto

\footnotetext{
Correspondencia:

Jaime A. Robledo, Corporación para Investigaciones Biológicas, Carrera 72A № 78B-141, Medellín, Colombia. Teléfono (574) 441 0855; fax: (574) 4415514. jrobledo@cib.org.co
}

Recibido: 15/08/03; aceptado: 16/02/04 costo, pueden estar fuera del alcance de la mayoría de los países en desarrollo, donde se localiza el 95\% de los casos de tuberculosis (4).

La American Thoracic Society ha recomendado incluir siempre un medio de cultivo sólido para todos los especímenes clínicos que se sospeche contengan Mycobacterium tuberculosis (5). Un medio de cultivo que genere un diagnóstico temprano, sensible, de fácil implementación, con posibilidad de realizar identificación presuntiva de especie de acuerdo con la morfología de la colonia y que sea económicamente accesible, sería una alternativa o complementaría los métodos tradicionales para el diagnóstico de tuberculosis.

En este contexto, nuestro grupo ha implementado y evaluado el medio $7 \mathrm{H} 11$ en capa delgada (CD7H11) como una opción diagnóstica para tuberculosis, mediante la visualización de microcolonias características del complejo $M$. tuberculosis (6) como una alternativa de bajo costo, rápida y confiable. En este trabajo se presentan los resultados de más de cinco años de experiencia con $\mathrm{CD} 7 \mathrm{H} 11$ en el diagnóstico rutinario de tuberculosis comparado con los medio de cultivo tradicionales.

\section{Materiales y métodos}

Entre enero de 1996 y abril de 2001 se recolectaron en la Unidad de Bacteriología y Micobacterias de la Corporación para Investigaciones Biológicas, 1.809 muestras clínicas de pacientes con sospecha clínica de tuberculosis. Estas muestras se procesaron de acuerdo con los procedimientos estándar para el diagnóstico microbiológico de tuberculosis $(5,7)$. Las muestras no estériles se trataron con $\mathrm{N}$-acetil-L-cisteína-hidróxido de sodio y se neutralizaron con solución tampón de fosfatos, $\mathrm{pH} \mathrm{6,6.} \mathrm{Todas} \mathrm{las} \mathrm{muestras} \mathrm{se}$ concentraron por centrifugación (3000 g) por 30 
minutos. Con el sedimento se realizó un extendido para ser coloreado con Kinyoun (7); se inoculó $0,1 \mathrm{ml}$ en el medio CD7H11 (en cajas de Petri plásticas de $90 \mathrm{~mm} \times 15 \mathrm{~mm}$ o de $60 \mathrm{~mm} \times 15$ $\mathrm{mm}$, selladas con cinta adhesiva pero permitiendo un centímetro de espacio para ventilación); 0,1 $\mathrm{ml}$ del mismo sedimento se inoculó en un tubo con medio Lowenstein-Jensen (LJ) y $0,1 \mathrm{ml}$ se inoculó en medio líquido Middlebrook 7H9. Las cajas con $\mathrm{CD} 7 \mathrm{H} 11$ se incubaron a $35^{\circ} \mathrm{C}$ en una atmósfera con $6 \%$ de $\mathrm{CO}_{2}$; los cultivos en LJ y los cultivos en $7 \mathrm{H} 9$ se incubaron en una atmósfera $\sin \mathrm{CO}_{2}$ a $35^{\circ} \mathrm{C}$. Estos últimos se incubaron por 15 días y, luego, se centrifugaron por 30 minutos a $3000 \mathrm{~g}$; del sedimento, se preparó un extendido para coloración de bacilos ácido-alcohol resistentes y se subcultivó en CD7H11. Los tubos con LJ y 7H9 se prepararon en nuestro laboratorio, según los procedimientos recomendados (7); los medios $\mathrm{CD} 7 \mathrm{H} 11$ se prepararon de acuerdo con los procedimientos descritos (6), con las siguientes modificaciones: la carbenicilina y la polimixina se reemplazaron por piperacilina $50 \mathrm{mg} / \mathrm{l}$ (la cual no es inhibitoria para el crecimiento de $M$. tuberculosis, probado en nuestro laboratorio antes de implementar su uso), anfotericina B y trimetoprim a las dosis recomendadas $(20 \mathrm{mg} / \mathrm{l})$. Todos los antibióticos se obtuvieron de una fuente comercial (Sigma Chemical Co., St. Louis, MO, USA).

Se cuantificaron los bacilos ácido-alcohol resistentes del extendido de 0 a cuatro cruces de acuerdo con los procedimientos recomendados (7). Las microcolonias características de $M$. tuberculosis en la capa delgada se identificaron por la morfología inicial, teniendo en cuenta la consistencia, los bordes de la colonia y la tendencia a formar cordones como se ha descrito tanto en medios líquidos como sólidos (6,8-10). La capa delgada se observó microscópicamente dos veces por semana durante un mes y el LJ se observó una vez por semana por dos meses. La identificación final de las colonias en ambos medios se realizó confirmando que estuvieran formadas por BAAR y en éstas se realizaron las pruebas de catalasa a $68^{\circ} \mathrm{C}$, reducción de nitratos a nitritos y de niacina (Difco Laboratories, Detroit, MI, USA) (7), esta última luego de ser subcultivado en LJ por cinco semanas. Todos los proce- dimientos se desarrollaron en una cámara de bioseguridad clase II.

Se evaluaron los cultivos obtenidos por LJ frente a los aislamientos en capa delgada según porcentaje de aislamientos, tiempo de detección y contaminación. Todos los grupos se compararon utilizando la prueba $t$ de Student o ji al cuadrado con $p<0,05$ como estadísticamente significativa. Se estimaron los indicadores de pruebas diagnósticas como sensibilidad, especificidad y valores predictivos con sus correspondientes intervalos de confianza del $95 \%$ y se excluyeron del análisis las muestras contaminadas en uno u otro medio. Se calculó el índice kappa para evaluar la concordancia entre ambas pruebas. Los crecimientos obtenidos también se compararon según la positividad del examen directo y el sitio de aislamiento, con un análisis semejante en ambos grupos.

\section{Resultados}

Entre enero de 1996 y abril de 2001 se recibieron y procesaron en el laboratorio diagnóstico de micobacterias 1.809 muestras clínicas provenientes de pacientes con diagnóstico clínico presuntivo de tuberculosis o de infección por micobacterias. De estas muestras, 41,6\% (752) eran de origen pulmonar y $58,4 \%$ (1.057) extrapulmonares.

El total de las muestras positivas por cualquier método fue de 170 (9,4\%); de éstas, 89 (52,4\%) eran pulmonares y $81(47,6 \%)$ extrapulmonares. Los aislamientos obtenidos de acuerdo con el tipo de muestras y el medio de cultivo empleado se muestran en el cuadro 1.

La mayoría de los aislamientos obtenidos se identificaron como $M$. tuberculosis $(86,4 \%)$, seguidos por Mycobacterium fortuitum (4,7\%) y Mycobacterium chelonae (2,9\%); Mycobacterium scrofulaceum y Mycobacterium avium se aislaron cada uno en el $1,8 \%$ de los casos. Como especies consideradas contaminantes sin importancia clínica, se aislaron Mycobacterium vaccae en $1,2 \%$ de las muestras, Mycobacterium flavescens y Mycobacterium smegmatis ambas en el $0,6 \%$ de ellas. Las especies de micobacterias no tuberculosas constituyeron el $13,6 \%$ de las 
Cuadro 1. Positividad total para Mycobacterium sp. según las muestras estudiadas y los medios de cultivo empleados.

\begin{tabular}{|c|c|c|c|c|c|}
\hline Muestra clínica & $\underset{n}{\text { Muestras }}$ & $\begin{array}{c}\text { Total positivos } \\
\mathbf{n} \\
(\%)\end{array}$ & $\begin{array}{c}\text { Positivos LJ } \\
\text { n } \quad(\%)\end{array}$ & $\begin{array}{c}\text { Positivos } \\
n\end{array}$ & $\begin{array}{l}\text { s CD7H11 } \\
(\%)\end{array}$ \\
\hline Esputo & 342 & $57(16,7)$ & $50 \quad(14,6)$ & 48( & $(14,0)$ \\
\hline Jugo gástrico & 159 & $1 \quad(0,6)$ & $(0,6)$ & 1 & $(0,6)$ \\
\hline Lavado broncoalveolar & 251 & $31 \quad(12,4)$ & $28 \quad(11,2)$ & 22 & $(8,8)$ \\
\hline Biopsias & 294 & $35 \quad(12,0)$ & $33 \quad(11,2)$ & 28 & $(9,5)$ \\
\hline Líquido pleural & 163 & $8 \quad(4,9)$ & $(4,9)$ & 4 & $(2,5)$ \\
\hline Líquido cefalorraquídeo & 247 & $15 \quad(6,1)$ & $(5,3)$ & 11 & $(4,5)$ \\
\hline Orina & 179 & (62) & $(5,6)$ & 5 & $(2,8)$ \\
\hline Líquido pericárdico & 39 & $(5,2)$ & $(5,2)$ & 2 & $(5,2)$ \\
\hline Pus & 66 & $8(12,1)$ & $8 \quad(12,1)$ & 4 & $(6,1)$ \\
\hline Otros $^{*}$ & 69 & $2 \quad(3,0)$ & $(3,0)$ & 1 & $(1,4)$ \\
\hline Total & 1.809 & $170 \quad(9,4)$ & $(8,6)$ & 126 & $(7,0)$ \\
\hline
\end{tabular}

* Dos muestras de líquido sinovial positivas

Cuadro 2. Sensibilidad, especificidad, valores predictivos y concordancia de CD7H11 en muestras pulmonares y extrapulmonares, tomando como referencia el cultivo en medio LJ.

\begin{tabular}{|c|c|c|c|c|c|}
\hline Muestras & $\begin{array}{c}\text { Sensibilidad } \\
\% \text { (IC95\%) }\end{array}$ & $\begin{array}{c}\text { Especificidad } \\
\%(\text { (IC95\%) }\end{array}$ & $\begin{array}{c}\text { VPP } \\
\%(\text { IC95\%) }\end{array}$ & $\begin{array}{c}\text { VPN } \\
\%(\text { (IC95\%) }\end{array}$ & $\begin{array}{c}\text { Coeficiente } \\
\text { kappa } \\
(I C 95 \%)\end{array}$ \\
\hline Pulmonares & $\begin{array}{c}81,0 \\
(72,4-89,7)\end{array}$ & $\begin{array}{c}98,9 \\
(98,2-99,7)\end{array}$ & $\begin{array}{c}90,1 \\
(83,2-97,1)\end{array}$ & $\begin{array}{c}97,8 \\
(96,7-98,9)\end{array}$ & $\begin{array}{c}0,58 \\
0,50-0,66\end{array}$ \\
\hline Extrapulmonares & $\begin{array}{c}65,8 \\
(55,1-76,5)\end{array}$ & $\begin{array}{c}99,5 \\
(99,0-99,9)\end{array}$ & $\begin{array}{c}90,9 \\
(83,3-98,5)\end{array}$ & $\begin{array}{c}97,4 \\
(96,4-98,4)\end{array}$ & $\begin{array}{c}0,46 \\
(0,36-0,55)\end{array}$ \\
\hline
\end{tabular}

especies aisladas. El 8,2\% (149/1.809) de los cultivos en $\mathrm{CD} 7 \mathrm{H} 11$ se contaminaron frente a $0,9 \%$ (17/1.809) de los cultivos en LJ.

El medio de cultivo LJ detectó 155 de 170 muestras positivas, comparado con 126 detectadas con CD7H11. Del total de muestras positivas, $41(24,1 \%)$ se detectaron solamente por LJ y $12(7,1 \%)$ por CD7H11, al excluir las muestras contaminadas en uno $u$ otro medio. Las muestras positivas en el examen directo fueron 85 (50\%); en ellas, la capa delgada alcanzó una proporción de detección de muestras positivas del $91,8 \%(78 / 85)$ y en LJ del $94,1 \%$ (80/85). El resto de las muestras (50\%) fueron negativas en el examen directo; se detectaron 48 (56,7\%) en CD7H11 y $75(88,2 \%)$ en LJ.

Se cultivaron concomitantemente 1.310 muestras en medio $7 \mathrm{H} 9$, de las cuales, 49 (3,74\%) fueron positivas, con 4 de estos aislamientos negativos en CD7H11 y en LJ.

Comparativamente con LJ, la sensibilidad para CD7H11 fue de 73,5\% (IC95\%: 69,6-80,4), la especificidad de 99,2\% (IC95\%: 98,8-99,6), el valor predictivo positivo de 90,4\% (IC95\%: 85,3$95,6)$ y el valor predictivo negativo de $97,6 \%$ (IC95\%: 96,8-98,3). La concordancia entre los dos métodos utilizando el coeficiente kappa fue de 0,52 (IC95\%: 0,46-0,58). La sensibilidad, la especificidad, los valores predictivos y la concordancia fueron mayores para CD7H11 cuando se analizaron las muestras de origen pulmonar, cuadro 2 .

El tiempo promedio para la detección de los crecimientos en capa delgada fue de $11 \pm 4,9$ días y de 26,5 58,6 días para los cultivos en LJ. Al final de la primera semana, los cultivos en CD7H11 lograron la detección de $65,3 \%$ de las muestras positivas frente a $1,3 \%$ de $\mathrm{LJ}$; para la segunda semana, la capa delgada había detectado $82,3 \%$ de las muestras positivas contra $14,6 \%$ de las muestras detectadas por LJ; en la tercera semana, el $95,2 \%$ de los cultivos fue positivo en capa delgada y el $40,4 \%$ de los cultivos en LJ. En la cuarta semana de observación, la capa delgada detectó el $100 \%$ de las micobacterias y LJ el 
$76,2 \%$. Las mayores diferencias en el tiempo al comparar la positividad de los dos métodos se observaron hasta la cuarta semana (figura 1).

Lowenstein-Jensen requirió en promedio 24,6+7,3 días para detectar las micobacterias que crecieron de muestras pulmonares y $28,5+9,3$ días para las provenientes de muestras extrapulmonares. El método de capa delgada utilizó en promedio $8,4 \pm 3,2$ días para las muestras pulmonares y $14 \pm 7,1$ días para las extrapulmonares. Los porcentajes de aislamientos para las muestras extrapulmonares y pulmonares según el medio de cultivo se muestran en la figura 2.

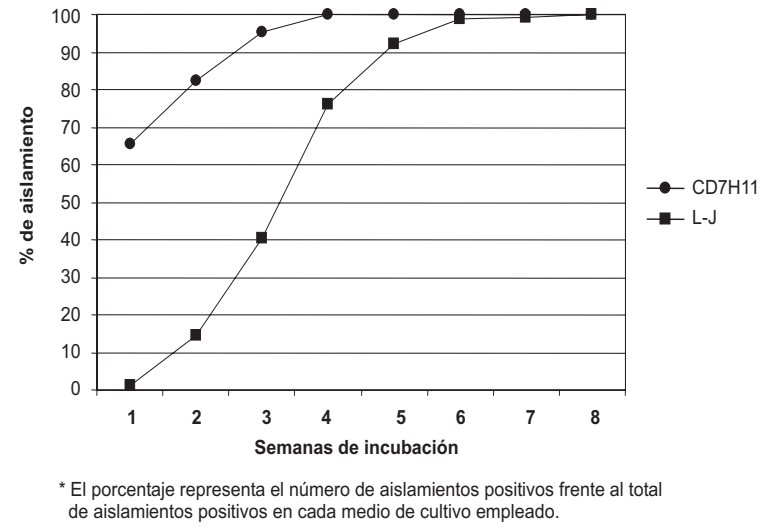

Figura 1. Tiempo requerido para el aislamiento de Mycobacterium sp. por cada medio de cultivo*.

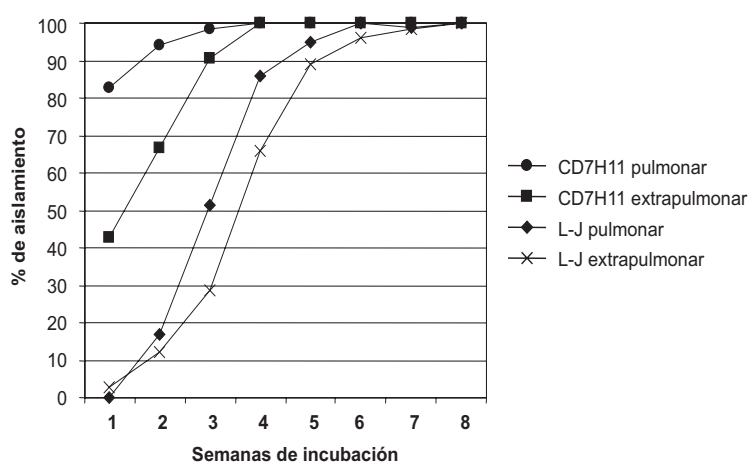

* El porcentaje representa el número de aislamientos positivos frente al total de aislamientos positivos en cada medio de cultivo empleado.

Figura 2. Tiempo necesario para obtener el crecimiento en cada medio de cultivo* de Mycobacterium sp. según el origen de la muestra.

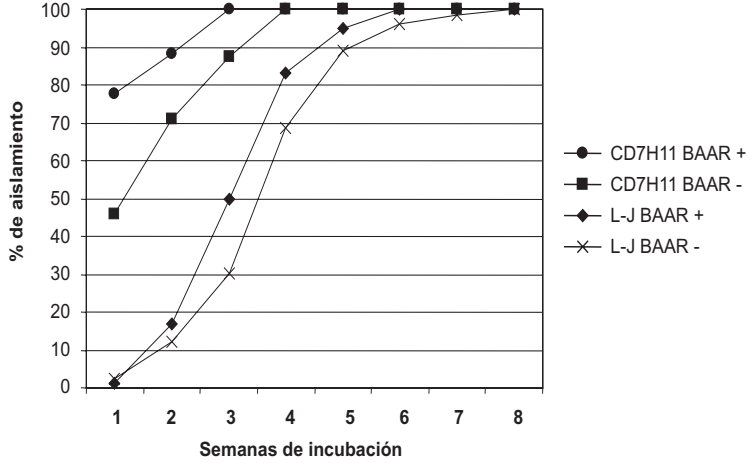

* El porcentaje representa el número de aislamientos positivos frente al total de aislamientos positivos en cada medio de cultivo empleado.

Figura 3. Tiempo necesario para obtener el crecimiento de Mycobacterium sp. según la positividad del examen directo*.

La capa delgada tardó en promedio 9,3 3 3,2 días para detectar los aislamientos con directo positivo y $13,7 \pm 6,8$ días para aquellos aislamientos con examen directo negativo. Los aislamientos en LJ se lograron, en promedio, a los $24,8 \pm 7,6$ días en las muestras con directo positivo y $28,3 \pm 9,1$ días en las muestras con examen directo negativo (figura 3).

En el medio CD7H11 crecieron $115(78,2 \%)$ de los aislamientos identificados como M. tuberculosis y $11(47,8 \%)$ de los aislamientos de micobacterias no tuberculosas. Lowenstein-Jensen detectó 136 $(92,5 \%)$ M. tuberculosis y $19(82,6 \%)$ de las no tuberculosas.

Todos los aislamientos identificados inicialmente por la morfología de las microcolonias en capa delgada como $M$. tuberculosis se confirmaron como $M$. tuberculosis por las pruebas bioquímicas convencionales.

\section{Discusión}

La disminución del tiempo requerido para el diagnóstico de tuberculosis es una de las estrategias planteadas para el mejor control de esta enfermedad. EI U.S. Departament of Health and Human Services (Healthy people 2010 Understanding and improving health), por ejemplo, ha propuesto como meta reducir el tiempo promedio en el laboratorio para confirmar y reportar los casos de tuberculosis a dos días para el $75 \%$ de éstos (11), lo que expresa la necesidad global de mejorar este diagnóstico. 
De acuerdo con lo anterior, han surgido múltiples técnicas en los últimos años, orientadas a mejorar la sensibilidad y la especificidad del examen directo, mejorar el tiempo para la detección y la sensibilidad de los métodos de cultivo y disminuir el tiempo necesario para su identificación.

Los métodos basados en técnicas de amplificación de ácidos nucleicos han disminuido el tiempo en reportar los resultados y mejorado notablemente la sensibilidad del examen directo en muestras del tracto respiratorio $(12,13)$ y en muestras extrapulmonares $(14,15)$. Sin embargo, aún no tienen amplia aplicación en laboratorios con bajos recursos debido a sus costos y a las exigencias técnicas y locativas que requieren.

El cultivo en capa delgada presentado en este estudio es un método que permite la detección más rápida en el crecimiento de micobacterias que el LJ, 11 y 26,5 días en promedio, respectivamente. Esto representa un diagnóstico por cultivo, aun en muestras paucibacilares, entre 12 a 15 días más temprano que los métodos de cultivo convencionales. Sólo el 7,2\% de los aislamientos en capa delgada, frente a $42,2 \%$ en LJ, se produjeron después de la tercera semana, periodo límite para considerar un aislamiento como rápido (12).

El tiempo de detección observado en la capa delgada es semejante al tiempo reportado para los medios de cultivo líquido y la detección radiométrica (Bactec 460 TB) y al reportado para los métodos de detección automatizada y no automatizada, no radiométricos (Bactec MGIT 960, MB/BacT system, MB Redox y ESP Culture System II) (16-22). La diferencia de la capa delgada con los métodos de cultivo anteriores radica en el costo y la complejidad de la tecnología empleada, además de la necesidad, en estos últimos, de hacer la identificación de especie una vez se detecte crecimiento. Para implementar la capa delgada no se requiere equipamiento adicional, solamente los elementos usuales en el laboratorio de diagnóstico de tuberculosis, incluso, el uso del microscopio convencional.

El método MODS (microscopic observation broth drug susceptibility) para el diagnóstico y las pruebas de sensibilidad a medicamentos de $M$. tuberculosis ha sido descrito como una alternativa rápida y de bajo costo (23). Este método está basado en el medio líquido de cultivo y la identificación de colonias características de $M$. tuberculosis. Los tiempos de detección informados para MODS (mediana de 9 días) son similares al promedio encontrado de días para capa delgada en el presente estudio, 11 días. Sin embargo, la sensibilidad reportada para MODS es mayor a la encontrada para la capa delgada y similar a la de los métodos automatizados como el MGIT (23). Estas diferencias en la sensibilidad pueden obedecer a varios aspectos: 1) la mayor parte de la población utilizada en el estudio de MODS, $78 \%$, fue multibacilar (examen directo positivo) frente al $50 \%$ de las estudiadas en el presente estudio; 2) todas las muestras utilizadas para MODS fueron esputos en comparación con el $52 \%$ de las estudiadas por capa delgada, y 3) está demostrado que los medios líquidos o la combinación de medios pueden tener una mayor sensibilidad que utilizar un solo medio de cultivo sólido $(12,24)$.

El periodo de observación de la capa delgada también pudiera influir en la sensibilidad del método. En el presente estudio se utilizó un tiempo de observación de 4 semanas para capa delgada en contraste con 8 semanas de observación para los cultivos de LJ. Esto debido a la utilización de una capa de agar delgado más susceptible a la desecación. En consecuencia, en las muestras con escaso número de bacilos, las colonias pudieran tomar más de 4 semanas en ser detectadas.

Una ventaja de utilizar el medio de capa delgada, al igual que el MODS, es que permite la identificación al mismo tiempo que detecta el crecimiento, debido a la morfología característica de las microcolonias. La tendencia a crecer, tanto en medio sólido como en líquido, formando madejas o cordones permite la identificación de M. tuberculosis (6,8-10,23). Los datos del presente estudio confirman lo anterior; todas las identificaciones iniciales de las microcolonias como $M$. tuberculosis fueron confirmadas posteriormente por pruebas bioquímicas convencionales. Según esto, es importante destacar que el uso de la capa delgada permitió 
la identificación temprana de $M$. tuberculosis en $56,7 \%$ de las muestras negativas al examen directo y en $65,8 \%$ de las muestras extrapulmonares.

La menor sensibilidad observada para la capa delgada frente al LJ Ileva a la recomendación de usarlo concomitantemente con otro medio de cultivo. El diagnóstico se beneficia de la rapidez de la capa delgada para detectar aislamientos, la identificación preliminar de $M$. tuberculosis por la morfología de la microcolonia y la sensibilidad del método de cultivo tradicional. El uso de dos medios de cultivo concomitantes también aumenta la capacidad del laboratorio para aislar micobacterias $(6,12,24)$; los datos de este estudio confirman lo anterior, $7,1 \%$ de los aislamientos se detectaron solamente en capa delgada y el $24,1 \%$ sólo en LJ.

La implementación de la capa delgada en el proceso diagnóstico de tuberculosis es particularmente útil en laboratorios con volumen de muestras bajo o medio, debido al tiempo que se requiere para la observación de las cajas, tal como se había sugerido en una publicación anterior (6). Su bajo costo y la facilidad de implementación lo convierten en una alternativa rápida para detectar $M$. tuberculosis frente a los métodos tradicionales de cultivo.

Por último, los métodos rápidos de diagnóstico de tuberculosis, como el de capa delgada presentado en este estudio, plantean la necesidad de ser evaluados bajo los aspectos de costobeneficio y costo-efectividad, tanto en los programas de salud pública como en el diagnóstico individual de los pacientes. La rapidez en diagnosticar un paciente con tuberculosis debe necesariamente repercutir favorablemente al disminuir la morbilidad y la mortalidad en el paciente y la transmisión de la enfermedad en la comunidad, si el tratamiento se hace en forma oportuna.

\section{Agradecimientos}

A Liliana Montoya del Instituto de Ciencias de la Salud, CES, por la ayuda con el análisis estadístico.

\section{Referencias}

1. World Health Organization. Report on infectious diseases. Removing obstacles to healthy developments. Geneve: World Health Organization; 1999.

2. Haas D. Mycobacterial diseases. En: Mandell G, Douglas $\mathrm{G}$, Benett J, editors. Principles and practice of infectious diseases. Fourth edition. New York: Churchill Livingstone; 1995. p.2213-43.

3. Drobniewski FA, Caws M, Gibson A, Young D. Modern laboratory diagnosis of tuberculosis. Lancet Infectious Disease 2003;3:141-7.

4. Sudre P, Ten Dam G, Chan C, Kochi A. Tuberculosis: a global overview of the situation today. Bull World Health Org 1992;70:149-59.

5. American Thoracic Society. Diagnostic standards and classification of tuberculosis in adults and children. Am J Respir Crit Care Med 2000;161:1376-95.

6. Mejía GI, Castrillón L, Trujillo H, Robledo J. Microcolony detection in $7 \mathrm{H} 11$ thin layer culture is an alternative for rapid diagnosis of Mycobacterium tuberculosis infection. Int J Tuberc Lung Dis 1999;3:13842.

7. Kent BD, Kubica GP. Public health micobacteriology. A guide for the level III laboratory. Atlanta: Centers for Diseases Control; 1985. p.36-9, 47-69, 185-7.

8. Welch DF, Guruswamy AP, Sides SJ, Charles HS, Gilchrist MJ. Timely culture for mycobacteria which utilizes a microcolony method. J Clin Microbiol 1993; 31:2178-84.

9. Yagupsky PV, Kaminski DA, Palmer KM, Nolte FS. Cord formation in Bactec $7 \mathrm{H} 12$ medium for rapid presumptive identification of Mycobacterium tuberculosis complex. J Clin Microbiol 1990;28:1451-3.

10. Runyon EH. Identification of mycobacterial pathogens utilizing colony characteristics. Am J Clin Pathol 1970; 54:578-86.

11. Hale YM, Pfyffer GE, Salfinger M. Laboratory diagnosis of mycobacterial infections: new tools and lessons learned. Clin Infect Dis 2001;33:834-46.

12. Tenover FC, Crawford JT, Huebner RE, Geiter LJ, Horsburgh CR, Good RC. The resurgence of tuberculosis: is your laboratory ready? J Clin Microbiol 1993;31:767-70.

13. Wang SX, Tay L. Evaluation of three nucleic acid amplification methods for direct detection of Mycobacterium tuberculosis complex in respiratory specimens. J Clin Microbiol 1999;37:1932-4.

14. Scarparo C, Piccoli P, Rigon A, Ruggiero G, Scagnelli M, Piersimoni C. Comparison of enhanced Mycobacterium tuberculosis amplified direct test with 
COBAS AMPLICOR Mycobacterium tuberculosis assay for direct detection of Mycobacterium tuberculosis complex in respiratory and extrapulmonary specimens. J Clin Microbiol 2000;38:1559-62.

15. Woods GL, Bergmann JS, Williams-Bouyer N. Clinical evaluation of the Gen-Probe amplified Mycobacterium tuberculosis direct test for rapid detection of Mycobacterium tuberculosis in select nonrespiratory specimens. J Clin Microbiol 2001;39: 747-9.

16. Roggenkamp A, Hornef MW, Masch A, Aigner B, Autenrieth IB, Heesemann J. Comparison of MB/BacT and BACTEC 460 TB systems for recovery of mycobacteria in a routine diagnostic laboratory. J Clin Microbiol 1999;37:3711-2.

17. Cambau E, Wichlacz C, Truffot-Pernot $\mathrm{CH}$, Jarlier V. Evaluation of the new MB Redox system for detection of growth of mycobacteria. J Clin Microbiol 1999;37: 2013-5.

18. Heifets L, Linder T, Sanchez T, Spencer D, Brennan. Two liquid medium systems, Micobacteria Growth Indicator Tube and MB Redox Tube, for Mycobacterium tuberculosis isolation from sputum specimens. J Clin Microbiol 2000;38:1227-30.

19. Alcaide F, Benítez MA, Escribá J, Martín R. Evaluation of the BACTEC MGIT 960 and the MB/BacT systems for recovery of mycobacteria from clinical specimens and for species identification by DNA AccuProbe. J Clin Microbiol 2000;38:398-401.

20. Somoskövi A, Ködmön C, Lantos A, Bártfai Z, Tamási L, Fuzy J, et al. Comparison of recoveries of Mycobacterium tuberculosis using the automated BACTEC MGIT 960 system, the BACTEC 460 system, and Lowenstein-Jensen medium. J Clin Microbiol 2000; 38:2395-7.

21. Williams-Bouyer N, Yorke R, Lee HI, Woods GL. Comparison of the BACTEC MGIT 960 and ESP Culture System II for growth and detection of mycobacteria. J Clin Microbiol 2000;38:4167-70.

22. Katila M-J, Katila P, Erkinjuntti-Pekkanen R. Accelerated detection and identification of mycobacteria with MGIT 960 and COBAS-AMPLICOR systems. J Clin Microbiol 1999;38:960-4.

23. Caviedes L, Lee TS, Gilman RH, Sheen P, Spellman E, Lee EH et al. Rapid, efficient detection and drug susceptibility testing of Mycobacterium tuberculosis in sputum by microscopic observation of broth cultures. J Clin Microbiol 2000;38:1203-8.

24. Cheng AF, Li MS, Chan CY, Lyon D, Wise R, Lee JC. Evaluation fo three culture media and their combination for the isolation of Mycobacterium tuberculosis from pleural aspirates of patients with tuberculous pleurisy. J Trop Med Hyg 1994;97:249-53.

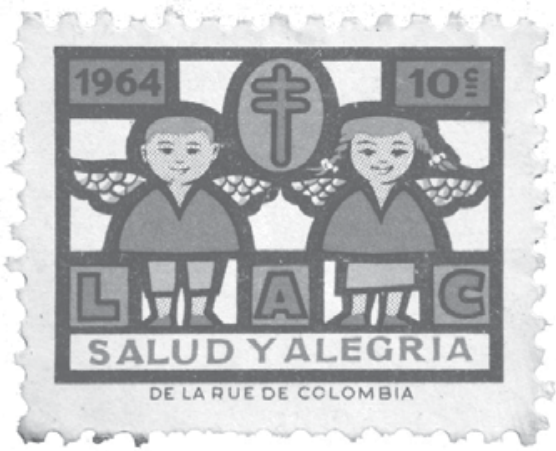

